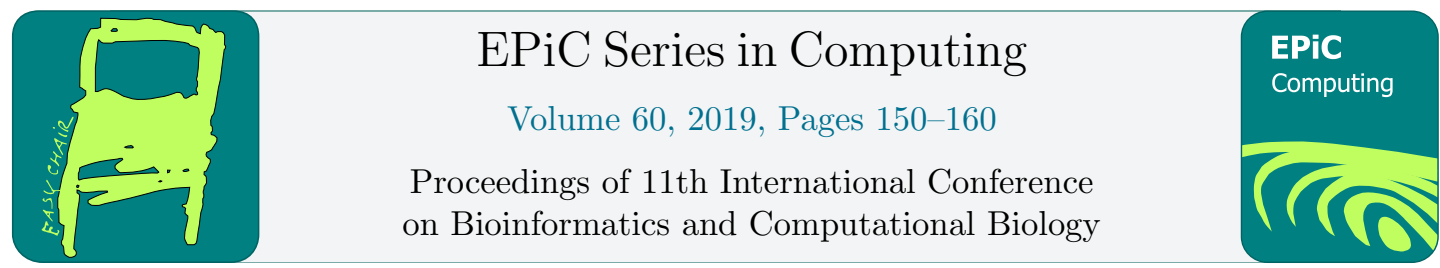

\title{
Petri nets and ODE as complementary tools in analysis of signaling pathways
}

\author{
Daria Kogut ${ }^{1}$, Kaja Gutowska ${ }^{2}$, Aleksandra Poterala-Hejmo ${ }^{1}$, Jaroslaw Smieja ${ }^{1}$, \\ Dorota Formanowicz ${ }^{3}$, and Piotr Formanowicz ${ }^{24}$ \\ ${ }^{1}$ Institute of Automatic Control, Silesian University of Technology, Gliwice, Poland \\ daria.kogut@polsl.pl, aleksandra.poterala-hejmo@polsl.pl, jaroslaw.smieja@polsl.pl \\ ${ }^{2}$ Institute of Computing Science, Poznan University of Technology, Poznan, Poland \\ Kaja.Gutowska@cs. put.poznan.pl, Piotr.Formanowicz@cs.put.poznan.pl \\ ${ }^{3}$ Department of Clinical Biochemistry and Laboratory Medicine, Poznan University of Medical \\ Sciences, Poznan, Poland \\ dof orman@ump.edu.pl \\ ${ }^{4}$ Institute of Bioorganic Chemistry, Polish Academy of Sciences, Poznan, Poland
}

\begin{abstract}
Regulation of gene expression is one of the most important problems analyzed in systems biology. It involves, among other, interactions of mRNA with miRNA - a small (21-25 nt) single-stranded non-coding RNA molecule. Its main function is post-transcriptional regulation of gene expression leading to gene silencing. It is achieved either by inhibition of translation or by degradation of mRNA. The detailed mechanisms employed include inhibition of attaching the 60s ribosomal subunit, premature ribosome drop-off or inhibition of protein elongation process, cleavage of mRNA or destabilization of mRNA. Another mechanism of regulation of gene expression involves reactive oxygen species (ROS - radical and non-radical oxygen species formed by the partial reduction of oxygen) which, being released from mitochondrium cytochrome $\mathrm{C}$ and inducing DNA damage, induce the apoptosis pathway. ROS level can be regulated by antioxidant systems existing in a cell. This paper presents analysis of a model of gene regulation based on these molecules, in which Petri net is used to find the key reactions and, subsequently, an ODE-based model is used to verify these conclusions.
\end{abstract}

\section{Introduction}

Proper functioning of the eukaryotic cells strongly depends on redox homeostasis, understood as a balance between production and elimination of reactive oxygen species (ROS). ROS are highly reactive molecules, containing oxygen, which are byproducts of aerobic respiration in mitochondria, activity of NADPH oxidases and several other enzymes. They are generated under physiological conditions as well as in response to xenobiotics, ionizing radiation or cytokines [13]. Moderate level of ROS is required for regulation of redox-sensitive proteins involved in proliferation and signal transduction [13]. Disruption of redox homeostasis, called oxidative stress, is related to the pathogenesis of various diseases, including cancer, Alzheimer's disease, 
Parkinson's disease, or diabetes (see, e.g., [7, 9]). Decreased level of ROS leads to impaired proliferation and immune functions, while overproduction results in damage of DNA, proteins, and lipids within the cell, and, in consequence, mutations and apoptosis [17].

To maintain redox balance, cells are equipped with several antioxidative mechanisms, including one based on glutathione. Glutathione is a low molecular weight thiol compound considered one of the most important antioxidants synthesized in cells, and the most abundant one [2]. This tripeptide, known also as $\gamma$-L-glutamyl-L-cysteinylglycine, is a product of a two-step reaction: addition of cysteine to glutamate, followed by the addition of glycine. The first, rate limiting, step is catalyzed by two proteins: glutamate-cysteine ligase catalytic subunit (GCLC) and glutamate-cysteine ligase modifier subunit (GCLM) forming a complex called GCL. Second reaction is catalyzed by glutathione synthetase (GSS) [8].

Glutathione exists in oxidized (GSSG) and reduced (GSH) form, and it is mainly accumulated in the cytoplasm (over 80\%) with the rest located in mitochondria, endoplasmic reticulum, and nucleus $[8,1]$. GSH is the main form, which can be used to reduce $\mathrm{H}_{2} \mathrm{O}_{2}$ into $\mathrm{H}_{2} \mathrm{O}$ and GSSG (reaction catalyzed by proteins from glutathione peroxidase (GPx) family) or spontaneously reacts with other radicals to neutralize them. Oxidized glutathione can be reduced back to GSH form either by glutathione reductase (GSR) or thioredoxin, and used again to eliminate ROS. GSH:GSSG ratio is an important indicator of oxidoreductive conditions and increased GSSG level serve as a marker of oxidative stress [1].

Another molecular specie, involved in the processes under investigation, is p53 protein, known best for its role in the regulation of cell death. Recently, it has been identified as a factor bidirectionally regulating redox environment (it serve as a pro- and antioxidant) [15]. Multiple elements associated with glutathione-based antioxidative pathway have been proved to be p53-dependent, including GCLC gene regulated at the transcription level by p53 and proteins regulated indirectly through the transcription factor NRF2 and miRNAs [11].

The aim of this work is to use two different methods for modeling and analysis of signaling pathways. The first method based on Petri net allows to create an interaction network with no quantitative parameters. Analysis of such model leads, among other, to determine the most significant subprocesses, or reactions, for the entire biological system. This approach allows to focus on selected issues in simulations of the second model based on differential equations.

\section{Methods}

\subsection{Petri net-based approach}

Formally a Petri net is defined as a 5 -tuple of the form: $Q=\left(P, T, F, W, M_{0}\right)$, where: $P=$ $\left\{p_{1}, \ldots, p_{n}\right\}$ is a finite set of places, $T=\left\{t_{1}, \ldots, t_{m}\right\}$ is a finite set of transitions, $F \subseteq(P \times T) \cup$ $(T \times P)$ is a finite set of arcs, $W: F \rightarrow Z^{+}$is a weight function, $M_{0}: P \rightarrow N$ is an initial marking, $P \cap T=\emptyset \wedge P \cup T \neq \emptyset[10]$.

The Petri net structure is a weighted directed bipartite graph, which consists of two disjoint subsets of vertices, called places and transitions, that can be connected by arcs. In graphical representation of Petri net places are depicted as circles and transitions as rectangles or squares. In biological context, places correspond to passive components, i.e., biological or chemical substrates and products, whereas transitions correspond to active components of the modeled system as chemical reactions. The quantities of passive components are represented by tokens (located in places and represented as dots). Tokens can flow from one place to another through transitions corresponding to the flow of information in the model. Moreover, the distribution of tokens corresponds to the state of the modeled system [10, 14]. Mentioned flow of tokens 
is governed by a transitions firing rule. A transition can be fired, when the number of tokens in each preceding place is equal or grater than the weight of the arc connecting the place with the transition $[4,10,16]$. The graphical representation of a Petri net helps to understand the complex network of dependencies, as well as its behavior, during simulation. However, it is a mathematical representation, called incidence matrix, that is crucial for the analysis. Such matrix $A$ is composed of $n$ rows, corresponding to places, and $m$ columns, corresponding to transitions. The entry $a_{i j}$ of matrix $A$ is an integer number, equal to the difference between the numbers of tokens present in place $p_{i}$ before and after firing transition $t_{j}$ [10]. The incidence matrix enables the determination of t-invariants, that are the basis for the analysis $[5,6]$. Such an invariant is a vector $x \in N^{m}$, which is a solution of equation $A \cdot x=0$, and it represents some subprocess occurring in the modeled system, which does not change its state. With every t-invariant $x$, there is associated set $\operatorname{supp}(x)=\left\{t_{j}: x_{j}>0, j=1,2 \ldots, m\right\}$, called its support. Firing every transition $t_{j} \in \operatorname{supp}(x) x_{j}$ times does not change the distribution of tokens in the net.

The analysis of t-invariants is based on searching for similarities between them, which in the biological context corresponds to the similarities between subprocesses. Each transition corresponds to a certain elementary process, hence searching for intersection of t-invariants supports is a key point of analysis. Transitions being elements of such intersection correspond to some elementary processes, which may be crucial for the modeled process. Moreover, they can also be a source of interesting, previously unknown properties of the analyzed biological system, because subprocesses containing such transitions can interact with each other.

Additionally, t-invariants can be grouped into t-clusters, while transitions can be grouped into MCT sets (Maximal Common Transition sets). Each such set contains transitions belonging to supports of exactly the same t-invariants. Both MCT sets and clusters correspond to certain functional blocks of the model system, for which biological meaning should be determined $[4,6,16]$. A more detailed description of the methods of analyzing models based on Petri net was described in [3].

\subsection{A pathway modeling with using differential equations}

Biology and medicine are increasingly using various technical devices and approaches to learn more and understand biochemical and physical processes behind cellular responses to stress factors and, ultimately, use this knowledge to treat diseases more effectively, including designing gene therapies or targeting particular signaling pathways. On the other hand, technology may also benefit from biological knowledge, as the latter provides inspiration for developing new methods to solve technical problems.

In this work, we present a particular regulatory system, involving ROS and miRNA species. Figure 1 shows the proposed diagram of the ROS level control pathway with glutathione. The sharp-pointed arrows represent stimulation of the process, to which the arrowhead is directed, and the blunt ends represent inhibition of the process. In the diagram two groups of compounds are distinguished - an oval is used to represent proteins, while the other shapes - other groups of compounds. The system has been described in two ways - by using the Petri net and by using differential equations. Differential equations describe the changes in the concentrations/quantities of molecules taken into account due to interactions between them.

We can build mathematical models in many other ways. In this article, we present two methods of modeling the ROS level control system: by using differential equations and by using Petri nets, of which this second method is innovative from the point of view of system biology. 


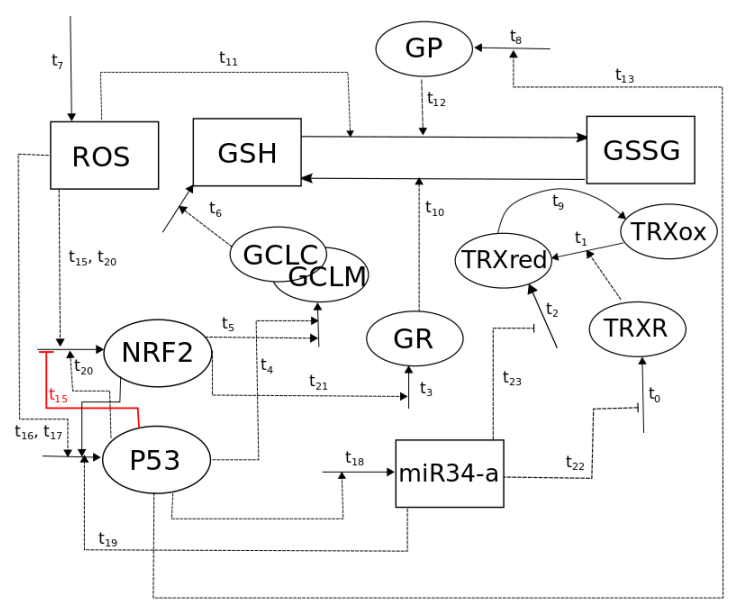

Figure 1: The proposed model of ROS regulation with glutathione.

\section{The Models}

Two models have been proposed as a part of the research described in this paper. The first one is based on the Petri net theory, while the other one is based on differential equations. The analysis of Petri net allows to determine which of the modeled subprocesses is the most important for the whole biological system, what in turn can be used in a model based on differential equations.

The proposed Petri net model of the biological system has been built using standalone Java application called Holmes [12]. The model shown in Figure 2 consists of several functional parts: 1.1) corresponding to relation between p53 and NRF2, 1.2) corresponding to relation between p53 and miR34a, 2.1) corresponding to oxidation-reduction reactions of GSH and GSSG, and 2.2) corresponding to oxidation-reduction reactions of $\mathrm{TRX}_{r e d}$ and $\mathrm{TRX}_{o x}$. The modeled system contains 4 non-trivial MCT sets, each containing more than one transition. Transitions belonging to these sets are marked in different colors. The names of all model components, both places and transitions, are included in Tables 1 and 2. The names of transitions from the Petri net based model, are contained in column called "Biological meaning (PN)", while column called "Biological meaning (ODE)" contains a description of coefficients in the differential equations model.

The proposed ODE model is a deterministic model based on mass-action law. This is a classic method of signaling pathway simulations. The equations were based on the scheme on Fig. 1. The model contains twelve equations, which represent concentration of each molecule in the system and their interactions with another molecules. The coefficients $\left(t_{i}\right)$ are describing reaction speed, which depends on another molecules. Output of the model represents concentration of molecules in stationary state. The results also show the number of compound molecules in the steady state after dimensionless time t.

Some simplifications appeared in the proposed models due to the limitations of the used methods. In Petri net model two different situations depending on environmental conditions have been proposed: the cytotoxic level of ROS and the physiological level of ROS. In both conditions, p53 protein is activated, however, depending on the conditions, it may inhibit or activate NRF2. In cytotoxic conditions p53 inhibits NRF2, while in physiological conditions p53 


$$
\begin{aligned}
\frac{\mathrm{d} R O S}{\mathrm{~d} t} & \left.=t_{7}-\left(t_{11} R O S(t)+t_{2} G P(t)\right) G S H(t)\right) \\
\frac{\mathrm{d} G S H}{\mathrm{~d} t} & =t_{6} G C L(t)+t_{10} G R(t) G S S G(t)-\left(t_{11} R O S(t)+t_{12} G P(t)\right) G S H(t) \\
\frac{\mathrm{d} G S S G}{\mathrm{~d} t} & =\left(t_{11} R O S(t)+t_{12} G P(t)\right) G S H(t)-t_{10} G R(t) G S S G(t) \\
\frac{\mathrm{d} G C L}{\mathrm{~d} t} & =t_{5} N R F 2(t)+t_{4} P 53(t) \\
\frac{\mathrm{d} N R F 2}{\mathrm{~d} t} & =t_{20} P 53(t)+t_{20} R O S(t) \\
\frac{\mathrm{d} P 53}{\mathrm{~d} t} & =t_{19} \operatorname{miR} 34 a(t)+t_{24} N R F 2(t)+t_{16} \operatorname{ROS}(t) \\
\frac{\mathrm{d} m i R 34 a}{\mathrm{~d} t} & =t_{18} P 53(t)-t_{22} m i R 34 a(t) \\
\frac{\mathrm{d} T R X_{R E D}}{\mathrm{~d} t} & =\frac{t_{2}}{t_{2}+m i R 34 a(t)}-t_{9} G S S G(t) T R X_{R E D}(t)+t_{1} T R X_{O X}(t) T R X R(t) \\
\frac{\mathrm{d} T R X_{R}}{\mathrm{~d} t} & =\frac{t_{0}}{t_{0}+m i R 34 a(t)} \\
\frac{\mathrm{d} T R X_{O X}}{\mathrm{~d} t} & =t_{9} T R X_{R E D} G S S G(t)-t_{1} T R X_{O X} T R X_{R}(t) \\
\frac{\mathrm{d} G R}{\mathrm{~d} t} & =t_{3} N R F 2(t) \\
\frac{\mathrm{d} G P}{\mathrm{~d} t} & =t_{8} P 53(t)
\end{aligned}
$$

activates NRF2. Additional simplification of the models is the positive feedback loop between p53 and miR34a. In the proposed models miR34a directly leads to activation of p53, however in a cell this is a more complex process, and not well explored.

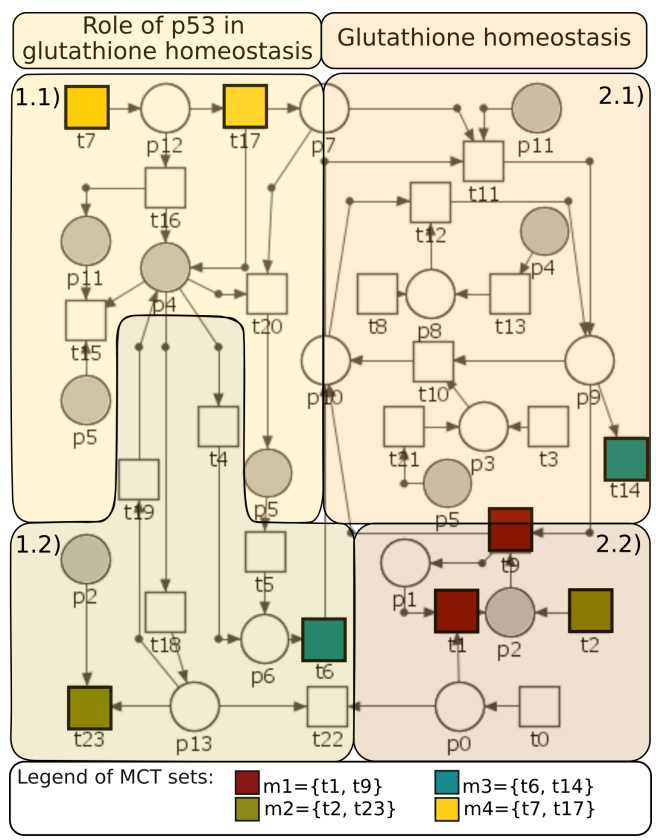

Figure 2: The proposed Petri net based model. 


\begin{tabular}{cccc}
\hline No. & Biological meaning & No. & Biological meaning \\
\hline$p_{0}$ & TRXR & $p_{7}$ & ROS \\
\hline$p_{1}$ & TRXox & $p_{8}$ & GP \\
\hline$p_{2}$ & TRXred & $p_{9}$ & GSSG \\
\hline$p_{3}$ & GR & $p_{10}$ & GSH \\
\hline$p_{4}$ & p53 & $p_{11}$ & more ROS \\
\hline$p_{5}$ & NRF2 & $p_{12}$ & pool of ROS \\
\hline$p_{6}$ & glutathione synthetases & $p_{13}$ & miR34a \\
\hline
\end{tabular}

Table 1: List of places

\section{Analyses and results}

The analysis of the Petri net model is mainly based on t-invariants whose number depends on the nature of the modeled system. The presented model consists of 14 places, 24 transitions, 4 non-trivial MCT sets and it is covered by 22 t-invariants. Biological meaning for MCT sets is included in Table 3.

Similar t-invariants are grouped into $12 \mathrm{t}$-clusters for which biological significance is described. The notification used in descriptions is as follows: $c_{12}=\left\{x_{21}, x_{22}\right\}$, where $c_{12}$ is a symbol of a cluster and $x_{21}, x_{22}$ are a symbols of t-invariants belonging to this cluster:

- $c_{1}=\left\{x_{1}\right\}$ : p53 activation influenced by cytotoxic ROS level leads to NRF2 inhibition, while p53 activation influenced by physiological ROS level leads to enhancement protein level of NRF2.

- $c_{2}=\left\{x_{2}\right\}$ : Positive feedback between p53 and miR34a (the model contains simplification, miR34a does not directly affect the enhancement of p53 activity).

- $c_{3}=\left\{x_{3}\right\}$ : GSH is oxidized to GSSG by GP, while reduction of GSSG to GSH occurs with simultaneous $\mathrm{TRX}_{r e d}$ oxidation to $\mathrm{TRX}_{o x}$.

- $c_{4}=\left\{x_{4}\right\}$ : GSH is oxidized to GSSG by GP and by ROS, while reduction of GSSG to GSH occurs with simultaneous $\mathrm{TRX}_{r e d}$ oxidation to $\mathrm{TRX}_{o x}$.

- $c_{5}=\left\{x_{5}, x_{9}, x_{13}, x_{17}\right\}:$ p53 activation influenced by physiological ROS levels leads to enhancement protein level of NRF2, which is involved in GSH synthesis by regulation of GCL activity. GSH is oxidized to GSSG by ROS, while reduction of GSSG to GSH by GR. Moreover, reduction to GSH occurs also with simultaneous $\mathrm{TRX}_{r e d}$ oxidation to $\mathrm{TRX}_{o x}$. Additionally, p53 has influence on transcription of miR34a, which inhibits $\mathrm{TRX}_{\text {red }}$.

- $c_{6}=\left\{x_{6}, x_{10}, x_{14}, x_{18}\right\}$ : This cluster contains all of the subprocesses described in cluster $c_{5}$, with one difference in which miR34a inhibits TRXR expression.

- $c_{7}=\left\{x_{7}\right\}$ : GSH oxidation to GSSG by GP, and reduction to GSH by GR.

- $c_{8}=\left\{x_{8}\right\}$ : GSH is oxidized to GSSG by GP (activation by p53) and by ROS, while reduction of GSSG to GSH is catalysed by GR.

- $c_{9}=\left\{x_{11}, x_{12}\right\}$ : GSH oxidation to GSSG by GP (which may be additionally activated by p53), and reduction of GSSG to GSH by GR. p53 activation influenced by physiological ROS level leads to enhancement protein level of NRF2, which modulates GSH redox state by GR regulation. 


\begin{tabular}{|c|c|c|}
\hline No. & Biological meaning (PN) & Biological meaning (ODE) \\
\hline$t_{0}$ & source of TRXR & production of TRXR \\
\hline$t_{1}$ & $\mathrm{TRX}_{o x}$ reduction to $\mathrm{TRX}_{r e d}$ & $\mathrm{TRX}_{o x}$ reduction to $\mathrm{TRX}_{\text {red }}$ \\
\hline$t_{2}$ & source of $\mathrm{TRX}_{\text {red }}$ & production of $\mathrm{TRX}_{r e d}$ \\
\hline$t_{3}$ & source of GR & GR production \\
\hline$t_{4}$ & $\begin{array}{c}\text { stimulation of GCL complex } \\
\text { expression }\end{array}$ & $\begin{array}{l}\text { stimulation of GCL complex } \\
\text { expression with P53 protein }\end{array}$ \\
\hline$t_{5}$ & $\begin{array}{c}\text { regulation of GCL complex } \\
\text { activity via NRF2 }\end{array}$ & $\begin{array}{c}\text { stimulation of GCL complex } \\
\text { activity via NRF2 }\end{array}$ \\
\hline$t_{6}$ & GSH synthesis & GSH synthesis \\
\hline$t_{7}$ & source of ROS production & ROS production \\
\hline$t_{8}$ & source of GP production & GP production \\
\hline$t_{9}$ & $\begin{array}{l}\text { reduction of GSSG to GSH } \\
\text { catalyzed with simultaneous } \\
\text { TRX }_{\text {red }} \text { oxidation to } \text { TRX }_{o x}\end{array}$ & $\begin{array}{c}\text { GSSG reduction to GSH } \\
\text { with simultaneous TRXred } \\
\text { oxidation to TRXox }\end{array}$ \\
\hline$t_{10}$ & $\begin{array}{c}\text { reduction of GSSG to GSH } \\
\text { catalyzed by GR }\end{array}$ & $\begin{array}{c}\text { reduction of GSSG to GSH } \\
\text { catalyzed by GR }\end{array}$ \\
\hline$t_{11}$ & $\begin{array}{c}\text { oxidation of GSH to GSSG } \\
\text { catalyzed by ROS }\end{array}$ & $\begin{array}{c}\text { oxidation of GSH to GSSG } \\
\text { catalyzed by ROS }\end{array}$ \\
\hline$t_{12}$ & $\begin{array}{c}\text { oxidation of GSH to GSSG } \\
\text { catalyzed by GP }\end{array}$ & $\begin{array}{c}\text { oxidation of GSH to GSSG } \\
\text { catalyzed by GP }\end{array}$ \\
\hline$t_{13}$ & activation of GP & $\begin{array}{c}\text { production of GP stimulated } \\
\text { with P53 protein }\end{array}$ \\
\hline$t_{14}$ & reduction of ROS level & - \\
\hline$t_{15}$ & NRF2 inhibition & $\begin{array}{l}\text { NRF2 inhibition with P53 } \\
\text { protein }\end{array}$ \\
\hline$t_{16}$ & $\begin{array}{l}\text { high ROS level and activation } \\
\text { of p53 influenced by cytotoxic } \\
\text { ROS level }\end{array}$ & $\begin{array}{c}\text { regulation of P53 production } \\
\text { with ROS }\end{array}$ \\
\hline$t_{17}$ & $\begin{array}{c}\text { low ROS level and activation } \\
\text { of p53 influenced by } \\
\text { physiological ROS level }\end{array}$ & $\begin{array}{c}\text { regulation of P53 production } \\
\text { with ROS }\end{array}$ \\
\hline$t_{18}$ & transcription of miR34a & transcription of miR34a \\
\hline$t_{19}$ & enhancing of p53 activity & $\begin{array}{c}\text { enhancing of p53 activity } \\
\text { with miR34a }\end{array}$ \\
\hline$t_{20}$ & $\begin{array}{c}\text { enhancing protein level } \\
\text { of NRF } 2 \text { by p } 53\end{array}$ & $\begin{array}{c}\text { enhancing protein level of } \\
\text { NRF } 2 \text { by p53 }\end{array}$ \\
\hline$t_{21}$ & $\begin{array}{c}\text { modulation of GSH redox } \\
\text { state by GR regulation }\end{array}$ & $\begin{array}{c}\text { stimulation production of } \\
\text { GR with NRF2 }\end{array}$ \\
\hline$t_{22}$ & inhibition of TRXR expression & inhibition of TRXR expression \\
\hline$t_{23}$ & inhibition of $\mathrm{TRX}_{r e d}$ & inhibition of TRXred \\
\hline
\end{tabular}

Table 2: List of transitions 


\begin{tabular}{cc}
\hline No. & Biological meaning \\
\hline$m_{1}$ & Thioredoxin redox reaction. \\
\hline$m_{2}$ & Inhibition of TRX red $_{\text {ra }}$ via miR34a. \\
\hline$m_{3}$ & GSH synthesis \\
\hline \multicolumn{3}{c}{ Pool of ROS (the model has certain simplification, therefore it contains 2 situations: } \\
cytotoxic level of ROS and physiological level of ROS which \\
are associated with particular places from model, \\
respectively $p_{11}$ - more ROS, and $p_{7}-$ ROS).
\end{tabular}

Table 3: List of MCT sets

- $c_{10}=\left\{x_{15}, x_{16}\right\}:$ p53 activation influenced by physiological ROS level enhances protein level of NRF2, which may influence on GSH synthesis via GCLC activity regulation. GSH is oxidized to GSSG through ROS and also by GP (which may be additionally activated by $\mathrm{p} 53)$.

- $c_{11}=\left\{x_{19}, x_{20}\right\}$ : Influence of p53 on GSH synthesis via regulation of GCLC expression. GSH is oxidized to GSSG through ROS and also by GP (which may be additionally activated by p53).

- $c_{12}=\left\{x_{21}, x_{22}\right\}$ : Influence of p53 on GSH synthesis via regulation of GCLC expression. GSH is oxidized to GSSG through ROS. Moreover, p53 is involved in transcription of miR34a, what inhibits TRX $_{r e d}$ and TRXR expression.

Analysis of t-invariants corresponding to the oxidation and reduction of glutathione showed the following frequencies:

- reaction of GSH oxidation to GSSG by ROS which represents about $73 \%$ of all subprocesses (16 out of 22 t-invariants),

- reaction of GSH oxidation to GSSG by GP which represents about $46 \%$ of all subprocesses (10 out of 22 t-invariants),

- reaction of GSSG reduction to GSH by GR which represents about $36 \%$ of all subprocesses (8 out of 22 t-invariants),

- reaction of GSSG reduction to GSH occurs with simultaneous TRX $\mathrm{T}_{\text {red }}$ oxidation to TRX $\mathrm{T}_{o x}$ which represents about $18 \%$ of all subprocesses ( 4 out of 22 t-invariants).

The ability to scavenge ROS in the modeled system is so significant that the subprocess associated with NRF2 inhibition (which is a consequence of activating p53 under conditions exhibiting high levels of ROS) occurs at a very low frequency of about $4 \%$ ( 1 of 22 t-invariants).

Knockout analysis allows to deactivate selected transitions (certain elementary process), what leads to the exclusion of some subprocesses (knockout analysis has been performed in Holmes [12]). Deactivation of transitions corresponding to GSH synthesis, disables all subprocesses related to ROS scavenging, to be precise 20 out of 22 t-invariants has been turned off. From the other two t-invariants, the first t-invariant corresponds to activation of p53 influenced by cytotoxic ROS level, what leads to NRF2 inhibition, while the second t-invariant corresponds to positive feedback between p53 and miR34a. Knockout analysis confirms that deactivation of a transition corresponding to GSH synthesis may lead to harmful accumulation of ROS. 
The results of t-invariants and knockout analysis were used to simulation of a model based on differential equations, which was focused on the reaction of GSH oxidation to GSSG by ROS. This reaction turned out to be the most significant in the proposed system. To validate whether this reaction plays a key role in the proposed model, simulations were carried out in two scenarios: in physiological conditions and with GSH knockout (a strong GSH degradation factor was inserted into the model). As the output we assumed ROS level due to the strongest relationships between those variables. The knowledge about this pathway allows to provide the result of the simulation, and confirm or deny the validity of the thesis, that knockout of GSH will disrupt the system.
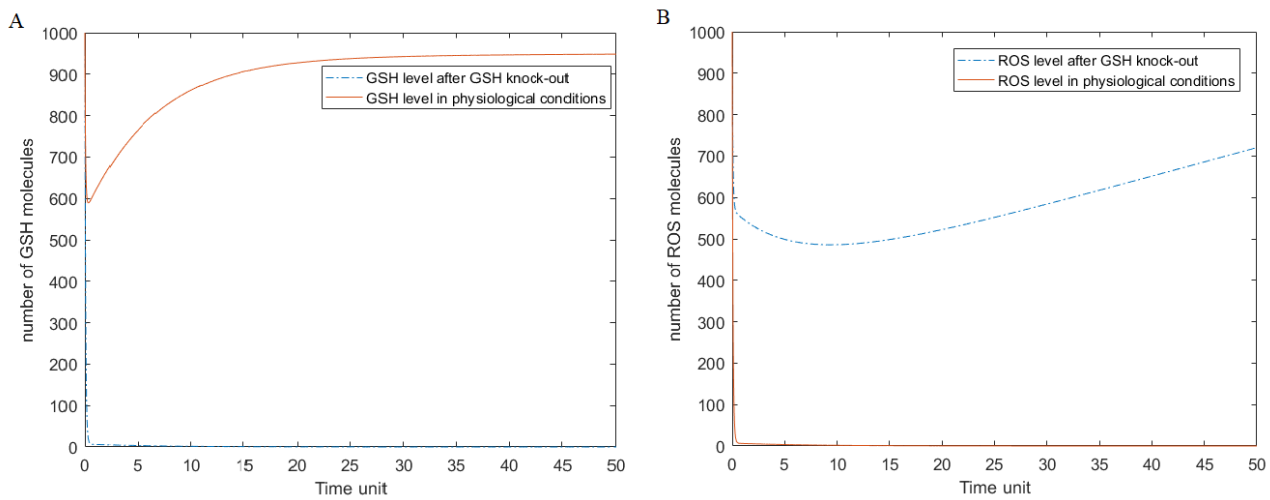

Figure 3: Results of the simulation of ODE model. The solid line represents level of ROS and GSH after GSH knockout, and the dotted line shows level of ROS and GSH in physiological conditions. Figure A shows the differences between GSH level before and after knockout; B represents ROS level before and after GSH knockout.

On Fig. 3 we can observe very different simulation results for each condition. For physiological condition, the ROS level is very low, because high level of GSH wiped out the ROS by oxidation processes. When the GSH is knockouted, the ROS level is rapidly increasing, because oxidation is the only way to remove reactive oxygen species from the system. We can conclude, that the knockout of glutathione can dysregulate the system, and lack of this dipeptide can lead to necrosis or apoptosis.

\section{Conclusions}

The model of controlling the ROS level in the system is a very useful, due to the fact that it presents how small imbalances can lead to system dysregulation. Often, biological knowledge is insufficient to determine unequivocally which reaction is the most significant for a given signaling pathway. Simultaneously, simulations of the model described by differential equations do not allow this type of analysis. Analysis of Petri net based model correctly indicated the reaction, which is confirmed by the results of simulations of differential equations. In conclusion, combining different methods to modeling and comparing them can be very useful and saves time looking for the main regulatory factors. The researches presented in paper are preliminary, and in further perspectives they will extend, especially in context of models. Petri net based models allow to discover previously unknown properties of the system. According to the presented 
results, discovered properties can be confirmed by simulations of model based on differential equations.

\section{Acknowledgments}

This work was funded by NCN grant DEC -2015/19/B/ST7/02984 and funded by BKM /RAU1/2018 (02/010/BKM18/0136).

\section{References}

[1] Nazzareno Ballatori, Suzanne M Krance, Sylvia Notenboom, Shujie Shi, Kim Tieu, and Christine L Hammond. Glutathione dysregulation and the etiology and progression of human diseases. Biological Chemistry, 390(3), 2009.

[2] Henry Jay Forman, Hongqiao Zhang, and Alessandra Rinna. Glutathione: overview of its protective roles, measurement, and biosynthesis. Molecular Aspects of Medicine, 30(1-2), 2009.

[3] Dorota Formanowicz, Kaja Gutowska, and Piotr Formanowicz. Theoretical studies on the engagement of interleukin 18 in the immuno-inflammatory processes underlying atherosclerosis. International Journal of Molecular Sciences, 19(11), 2018.

[4] Dorota Formanowicz, Adam Kozak, Tomasz Głowacki, Marcin Radom, and Piotr Formanowicz. Hemojuvelin-hepcidin axis modeled and analyzed using petri nets. Journal of Biomedical Informatics, 46(6), 2013.

[5] Eva Grafahrend-Belau, Falk Schreiber, Monika Heiner, Andrea Sackmann, Björn H Junker, Stefanie Grunwald, Astrid Speer, Katja Winder, and Ina Koch. Modularization of biochemical networks based on classification of petri net t-invariants. BMC Bioinformatics, 9(1), 2008.

[6] Ina Koch, Wolfgang Reisig, and Falk Schreiber. Modeling in systems biology: the Petri net approach, volume 16. Springer Science \& Business Media, 2010.

[7] Geou-Yarh Liou and Peter Storz. Reactive oxygen species in cancer. Free Radical Research, 44(5), 2010.

[8] Shelly C Lu. Glutathione synthesis. Biochimica et Biophysica Acta (BBA)-General Subjects, 1830(5), 2013.

[9] Shanmugam Manoharan, Gilles J Guillemin, Rajagopal Selladurai Abiramasundari, Musthafa Mohamed Essa, Mohammed Akbar, and Mohammed D Akbar. The role of reactive oxygen species in the pathogenesis of alzheimer's disease, parkinson's disease, and huntington's disease: a mini review. Oxidative Medicine and Cellular Longevity, 2016.

[10] Tadao Murata. Petri nets: Properties, analysis and applications. Proceedings of the IEEE, 77(4), 1989.

[11] Francisco Navarro and Judy Lieberman. mir-34 and p53: new insights into a complex functional relationship. PloS One, 10(7), 2015.

[12] Marcin Radom, Agnieszka Rybarczyk, Bartłomiej Szawulak, Hubert Andrzejewski, Piotr Chabelski, Adam Kozak, and Piotr Formanowicz. Holmes: a graphical tool for development, simulation and analysis of petri net based models of complex biological systems. Bioinformatics, 33(23), 2017.

[13] Paul D Ray, Bo-Wen Huang, and Yoshiaki Tsuji. Reactive oxygen species (ros) homeostasis and redox regulation in cellular signaling. Cellular Signalling, 24(5), 2012.

[14] Wolfgang Reisig. Understanding petri nets: modeling techniques, analysis methods, case studies. Springer, 2013.

[15] Anna A Sablina, Andrei V Budanov, Galina V Ilyinskaya, Larissa S Agapova, Julia E Kravchenko, and Peter M Chumakov. The antioxidant function of the p53 tumor suppressor. Nature Medicine, 11(12), 2005. 
[16] Andrea Sackmann, Monika Heiner, and Ina Koch. Application of petri net based analysis techniques to signal transduction pathways. BMC Bioinformatics, 7(1), 2006.

[17] Michael Schieber and Navdeep S Chandel. Ros function in redox signaling and oxidative stress. Current Biology, 24(10), 2014. 